\title{
Charmed-Meson Decay Constants from Improved QCD Sum Rules
}

\author{
Wolfgang Lucha* \\ Institute for High Energy Physics, Austrian Academy of Sciences, Nikolsdorfergasse 18, A-1050 \\ Vienna, Austria \\ E-mail: Wolfgang. Lucha@oeaw.ac.at
}

\section{Dmitri Melikhov}

Institute for High Energy Physics, Austrian Academy of Sciences, Nikolsdorfergasse 18, A-1050

Vienna, Austria,

Faculty of Physics, University of Vienna, Boltzmanngasse 5, A-1090 Vienna, Austria, and

D. V. Skobeltsyn Institute of Nuclear Physics, Moscow State University, 119991, Moscow, Russia

E-mail: dmitri_melikhovegmx.de

\section{Silvano Simula}

INFN, Sezione di Roma Tre, Via della Vasca Navale 84, I-00146 Roma, Italy

E-mail: simula@roma3.infn.it

The decay constants $f_{D_{(s)}}$ of the charmed heavy pseudoscalar mesons $D$ and $D_{s}$ are revisited within a recently developed novel approach to dispersive QCD sum rules which relies on an unprejudiced implementation of quark-hadron duality. The proposed modification of the conventional sum-rule techniques is assessed by applying our prescriptions to quantum mechanics, where exact solutions may be easily obtained by simply solving the Schrödinger equation. The very striking similarity of the extraction procedures of bound-state parameters in potential models and in QCD gives us great confidence in the reliability of our improvements of the sum-rule formalism and their applicability to hadron phenomenology. The implications of one's chosen definition of the heavy-quark masses are scrutinized and the $\overline{\mathrm{MS}}$ quark-mass scheme is identified as the optimal choice for our purposes. Our ideas turn out to reconcile QCD sum-rule predictions for the charmed-meson decay constants, which before tended to be markedly too low, with the findings of both lattice QCD and experiment.

The 2011 Europhysics Conference on High Energy Physics - HEP 2011,

July 21-27, 2011

Grenoble, Rhône-Alpes, France

\footnotetext{
${ }^{*}$ Speaker.
} 


\section{Introduction: Improvement of QCD Sum Rules as Incentive and Main Aim [1 -5]}

Unlike lattice gauge theories, QCD sum rules allow for fully analytic investigations of hadrons: $Q C D$ sum rules relate hadronic features to QCD by evaluation of matrix elements of suitably chosen operators on both hadron and QCD level and by assuming quark-hadron duality: above an effective threshold the perturbative QCD contribution cancels the one of hadronic excitations and continuum. Regarding the threshold as function of a parameter entering upon Borel transformation, we improve this concept by straightforward simple techniques [1 - 3] enabling us to estimate intrinsic errors too.

\section{Nonrelativistic Potential Models in Quantum Mechanics as Test Area of our Ideas}

The lack of a clear route to all their errors is a not unsurmountable weakness of QCD sum rules: Modelling the strong interactions by the funnel potential (or the like) describing heavy-quark bound states [6], we demonstrate [4] the capability of our step by application to quantum mechanics. There the exact bound-state features can be derived by numerical solution [7] of the Schrödinger equation. Requiring the duality-truncated QCD member of the sum rule to counterbalance exactly its hadronic counterpart, that is, the lowest hadronic term, we prove that the induced exact threshold depends not only on the external momenta involved but also on the Borel parameter and the underlying operator.

\section{Decay Constants $f_{D}$ and $f_{D_{s}}$ of the Charmed Pseudoscalar Mesons $D$ and $D_{s}$ [5]}

Procedural resemblances then justify to apply our concepts for alteration also to real-life QCD: In hadron phenomenology, the behaviour of the effective threshold may be fixed by fitting predicted ground-state observables, for instance, hadron masses, to their experimentally observed values. The decay constant $f_{P}$ of a pseudoscalar meson $P \equiv(Q \bar{q})$ of mass $M_{P}$, composed of a heavy quark $Q$ and a light quark $\bar{q}$, is defined by $\left(m_{Q}+m_{q}\right)\left\langle 0\left|\bar{q} \mathrm{i} \gamma_{5} Q\right| P\right\rangle=f_{P} M_{P}^{2}$. With $\bar{m}_{c}\left(\bar{m}_{c}\right)=(1.279 \pm 0.013) \mathrm{GeV}$ for the $\overline{\mathrm{MS}} c$-quark mass, the outcome of our approach for the decay constants of the charmed $D$ and $D_{s}$ mesons exhibits a perfect agreement with the findings of lattice gauge theory and experiment [5]: $f_{D}=\left(206.2 \pm 7.3_{\mathrm{QCD}} \pm 5.1_{\text {syst }}\right) \mathrm{MeV}$ and $f_{D_{s}}=\left(245.3 \pm 15.7_{\mathrm{QCD}} \pm 4.5_{\text {syst }}\right) \mathrm{MeV}$, where the QCD error comprises all uncertainties of the various (perturbative and nonperturbative) QCD parameters.

\section{Brief Summary: Observations, Findings, Results, Conclusions, and Outlook $[4,5]$}

Clearly, the QCD sum-rule formalism offers plenty of room for increasing its predictive power:

- The exact effective continuum thresholds do depend on both Borel parameter and the relevant momenta; moreover, they are not universal (that is, they vary with the correlator under study).

- As a whole, our proposed modifications and improvements raise dramatically the accuracy of the traditional sum-rule predictions and yield tenable estimates of their intrinsic uncertainties.

- The blatant quantitative similarity of our hadron-parameter extraction procedures in potential models and in QCD calls for QCD applications, e.g., to pseudoscalar-meson form factors [8].

- Our sophisticated approach reconciles QCD sum rules and experiment (as depicted in Fig. 1). 

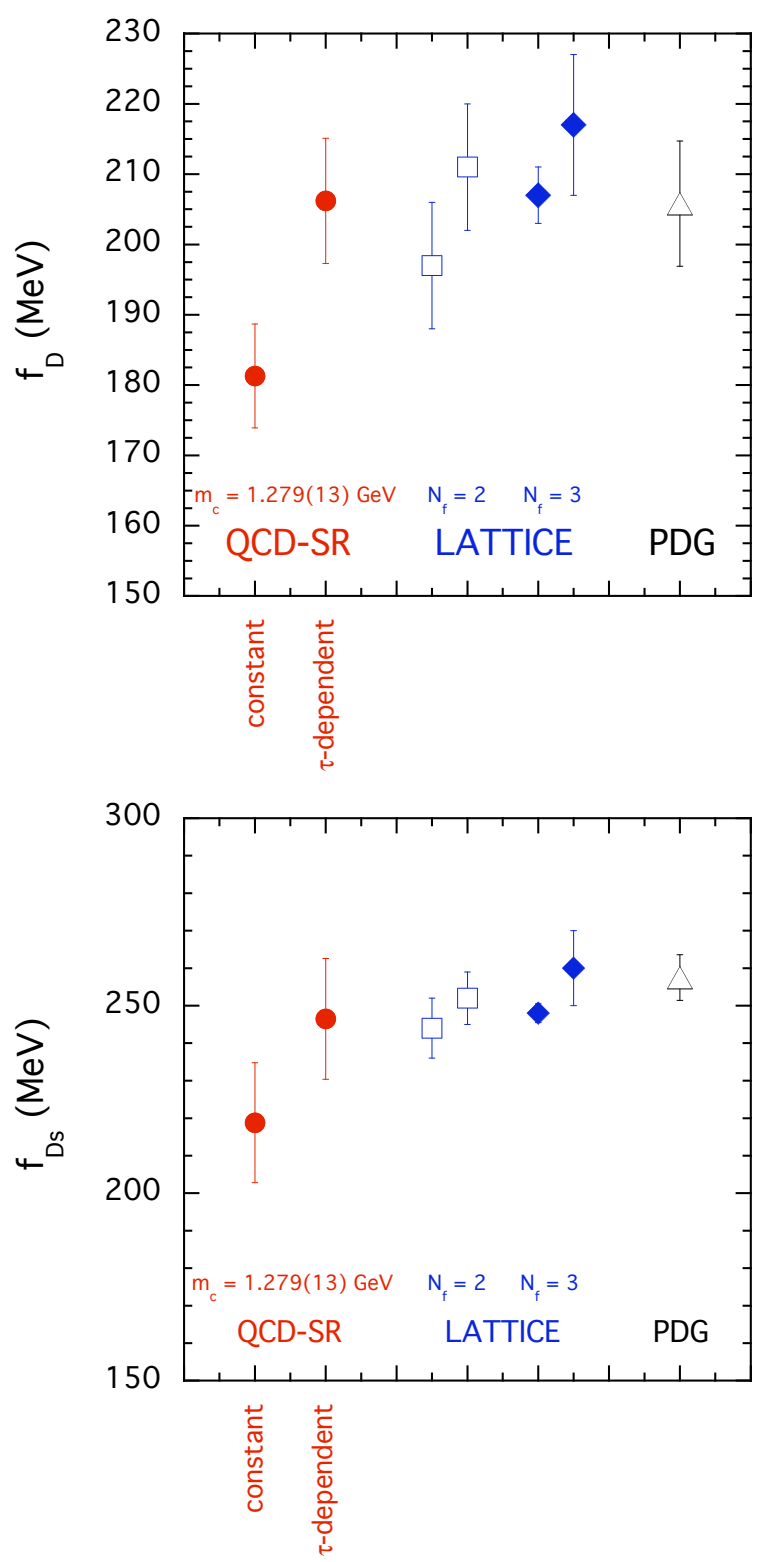

Figure 1: The dependence of the effective thresholds on the Borel parameter $\tau$ visibly improves the sum-rule results for the decay constants $f_{D_{(s)}}$, as a comparison with the findings of lattice QCD and experiment reveals.

\section{Acknowledgments}

D.M. is grateful for financial support by the Austrian Science Fund (FWF), project no. P22843.

\section{References}

[1] W. Lucha, D. Melikhov, and S. Simula, Phys. Rev. D 76 (2007) 036002; in QCD@Work 2007, eds. P. Colangelo et al., AIP Conf. Proc. 964 (AIP, Melville, New York, 2007), p. 296; Phys. Atom. Nucl. 71 (2008) 1461; Phys. Lett. B 657 (2007) 148; in Hadron 07, eds. L. Benussi et al., Frascati Phys. Ser. 46 (INFN, Laboratori Nazionali di Frascati, 2007), p. 1109; PoS Confinement8 (2009) 180. 
[2] W. Lucha, D. Melikhov, and S. Simula, Phys. Lett. B 671 (2009) 445; PoS Confinement8 (2009) 106; D. Melikhov, Phys. Lett. B 671 (2009) 450; arXiv:1112.2157 [hep-ph].

[3] W. Lucha, D. Melikhov, and S. Simula, Phys. Rev. D 79 (2009) 096011; J. Phys. G: Nucl. Part. Phys. 37 (2010) 035003; W. Lucha, D. Melikhov, H. Sazdjian, and S. Simula, Phys. Rev. D 80 (2009) 114028.

[4] W. Lucha, D. Melikhov, and S. Simula, Phys. Lett. B 687 (2010) 48; Phys. Atom. Nucl. 73 (2010) 1770; in QCD@Work 2010, eds. L. Angelini et al., AIP Conf. Proc. 1317 (AIP, Melville, New York, 2010), p. 316; in QCHS IX, eds. F. J. Llanes-Estrada and J. R. Peláez, AIP Conf. Proc. 1343 (AIP, Melville, New York, 2011), p. 624; in Hadron 2011, eds. B. Grube et al., eConf C110613 (2011), p. 841 .

[5] W. Lucha, D. Melikhov, and S. Simula, in QCD@Work 2010, eds. L. Angelini et al., AIP Conf. Proc. 1317 (AIP, Melville, New York, 2010), p. 310; PoS ICHEP 2010 (2010) 210; J. Phys. G: Nucl. Part. Phys. 38 (2011) 105002; in QCHS IX, eds. F. J. Llanes-Estrada and J. R. Peláez, AIP Conf. Proc. 1343 (AIP, Melville, New York, 2011), p. 379; PoS QFTHEP2010 (2010) 058; Phys. Lett. B 701 (2011) 82; in Hadron 2011, eds. B. Grube et al., eConf C110613 (2011), p. 584; PoS EPS-HEP2011 (2012) 153.

[6] W. Lucha, F. F. Schöberl, and D. Gromes, Phys. Rep. 200 (1991) 127; W. Lucha and F. F. Schöberl, Int. J. Mod. Phys. A 7 (1992) 6431.

[7] W. Lucha and F. F. Schöberl, Int. J. Mod. Phys. C 10 (1999) 607.

[8] V. Braguta, W. Lucha, and D. Melikhov, Phys. Lett. B 661 (2008) 354; W. Lucha and D. Melikhov, PoS Confinement8 (2009) 072; arXiv:1110.2080 [hep-ph], J. Phys. G: Nucl. Part. Phys. 39 (2012) (in press); I. Balakireva, W. Lucha, and D. Melikhov, in QCHS IX, eds. F. J. Llanes-Estrada and J. R. Peláez, AIP Conf. Proc. 1343 (AIP, Melville, New York, 2011), p. 615; arXiv:1103.3781 [hep-ph]; in Hadron 2011, eds. B. Grube et al., eConf C110613 (2011), p. 265; PoS EPS-HEP2011 (2012) 305; arXiv:1110.6904 [hep-ph], Phys. Rev. D 85 (2012) (in press); PoS QFTHEP2011 (2012) 056; I. Balakireva, PoS QFTHEP2010 (2010) 059. 